\title{
Tailgut Cyst in a Neonate: A Case Report
}

\author{
Ji Hyen Lee · Yun Suk Lee · So Yeon Shim · Su Jin Cho · Eun Ae Park · Soon Sup Chung ${ }^{1}$. Sanghui Park ${ }^{2}$ \\ Departments of Pediatrics, ${ }^{1}$ Surgery, and ${ }^{2}$ Pathology, Ewha Womans University School of Medicine, Seoul, Korea
}

A tailgut cyst is a congenital anomaly located in the retrorectal space. This cyst is usually detected in adult patients, and is rarely diagnosed in the neonatal period., ${ }^{1,2}$ These retrorectal cystic masses are believed to originate from the embryonic hindgut. ${ }^{1,3}$ Following a literature review, we report a case of tailgut cyst in a neonate that was removed entirely through surgical excision.

\section{CASE REPORT}

A $3.3-\mathrm{kg}$ baby girl was born at 37 weeks gestation by Cesarean section. During prenatal ultrasonography, a large mass was found in the pre-sacrococcygeal space. Local examination showed a $4 \times 5$-cm-sized swelling in the gluteal region, and laboratory tests were within normal limits. An X-ray of the lumbosacral spine indicated a soft mass in the coccygeal area without calcification (Fig. 1A). Ultrasonography showed a multiloculated cystic lesion with some echogenic portions at the pelvis that were located lateral and posterior to the rectosigmoid colon and anterior to the sacrococcygeal area (Fig. 1B). Magnetic resonance imaging (MRI) was performed and showed a large, well-demarcated mass with a small septated cyst within. There were calcifications within the upper portion of the cystic mass (Fig. 1C). When the infant was five days old, given concern for a possible sacrococcygeal cystic teratoma, a complete excision up to the coccyx was done through a posterior approach. The histopathology report showed a well-circumscribed, gray, soft mass $(4.7 \times 4.5 \times$ $3.5 \mathrm{~cm})$ with an attached ellipse of skin $(2.3 \times 1.9 \mathrm{~cm})$, and the

\section{Corresponding Author}

Eun Ae Park, MD

Department of Pediatrics, Ewha Womans University School of Medicine, 1071 Anyangcheon-ro, Yangcheon-gu, Seoul 07985, Korea

Tel: +82-2-2650-5574, Fax: +82-2-2650-3718, E-mail: pea8639@ewha.ac.kr

Received: October 16, 2015 Revised: November 25, 2015

Accepted: November 27, 2015 cut section revealed a multilocular cyst. Microscopically, the cyst walls were lined by various epithelia: mucinous, low cuboidal, and pseudostratified columnar cells (Fig. 2). Additionally, dotted bundles of smooth muscle fibers and fibrous tissues were present between the cystic walls. No specific tissue fragments of significance such as skin adnexal elements, neural elements, or heterologous mesenchymal tissue (e.g., cartilage or bone) were found. The patient was discharged after 12 days and followed up as an outpatient with no postoperative complications.

This study was approved by the Institutional Review Board (IRB) of Ewha Womans University Mokdong Hospital (IRB No. 2015-08-002).

\section{DISCUSSION}

Tailgut cysts are uncommon congenital masses in the neonate, and they are commonly located in the retrorectal space; embryonically, they are believed to arise from vestigial remnants of the embryonic hindgut. Normally, at 35 days of gestation, the embryo has a true tail during early human development that reaches a maximum of $8 \mathrm{~mm}$ in length. ${ }^{1}$ Theprimitive hindgut extends into this embryonic true tail, which is located at the site of the formation of the anus. During the eighth week of embryonic development, the tailgut usually atrophies, but occasionally can fail to regress completely. Some investigators have suggested that these remnants give rise to tailgut cysts. ${ }^{1}$

Tailgut cysts are more common in middle-aged women, and neonatal cases are considerably rare. The cyst usually occurs between the ages of 4 to 73 years, with an average presentation at 35 years, and the female-to-male ratio is 3:1. ${ }^{3}$ Typical symptoms of tailgut cysts are closely associated with their region. Most adult patients complain of lower back pain or rectal pain while sitting or after falling on their buttocks, and other symptoms include urinary retention, dysuria, changes in stool caliber, and rec- 
tal bleeding.

Developmental cysts can be classified according to their morphology and pathological features into sacrococcygeal teratomas, epidermoid cysts, dermoid cysts, enteric duplication cysts, gland cysts, anterior meningocele, and tailgut cysts. ${ }^{1,2}$ Sacrococcygeal teratomas are the most common mass in newborns and consists of mixed elements of three germ cell layers, such as skin adnexa and neural elements. Epidermoid cysts are lined by a stratified squamous epithelium with lack smooth muscle fibers in the walls. In our study, skin appendages such as hair follicles and
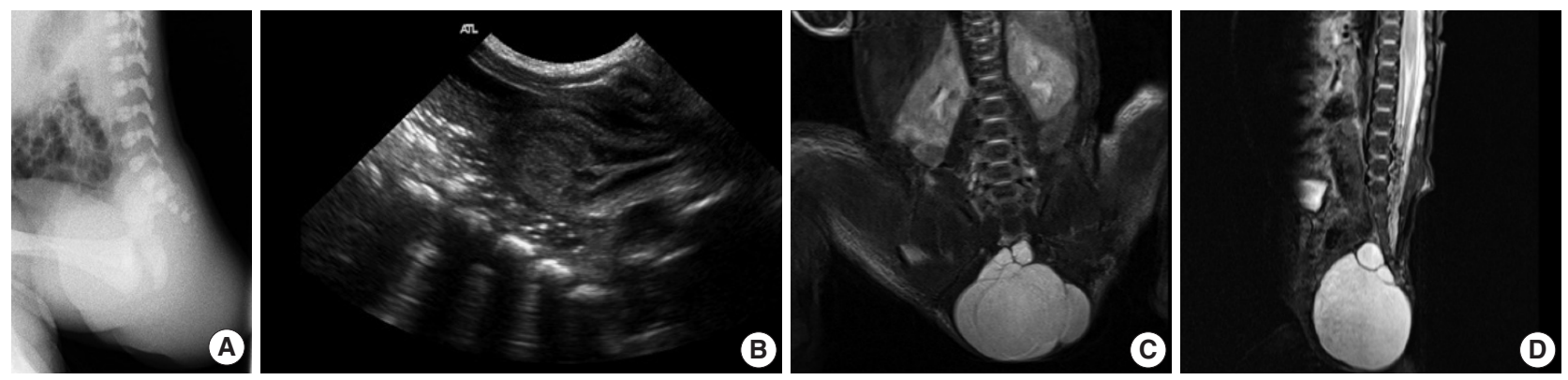

Fig. 1. Lumbosacral spinal X-ray shows a soft mass in the coccygeal area without calcification. (A) The cystic mass in the sacococcygeal area with intrapelvic and extrapelvic components (type II) is shown by abdominal sonography. (B) Sagittal magnetic resonance image shows a large cystic mass $(6.2 \times 4.2 \times 5.6 \mathrm{~cm})$ with a few small septae. This cystic mass is shown with low signal intensity in a T1W1 image and high signal intensity in a T2W1 image. (C, D) There are calcifications within the upper portion of the cystic mass with dark signal intensity.
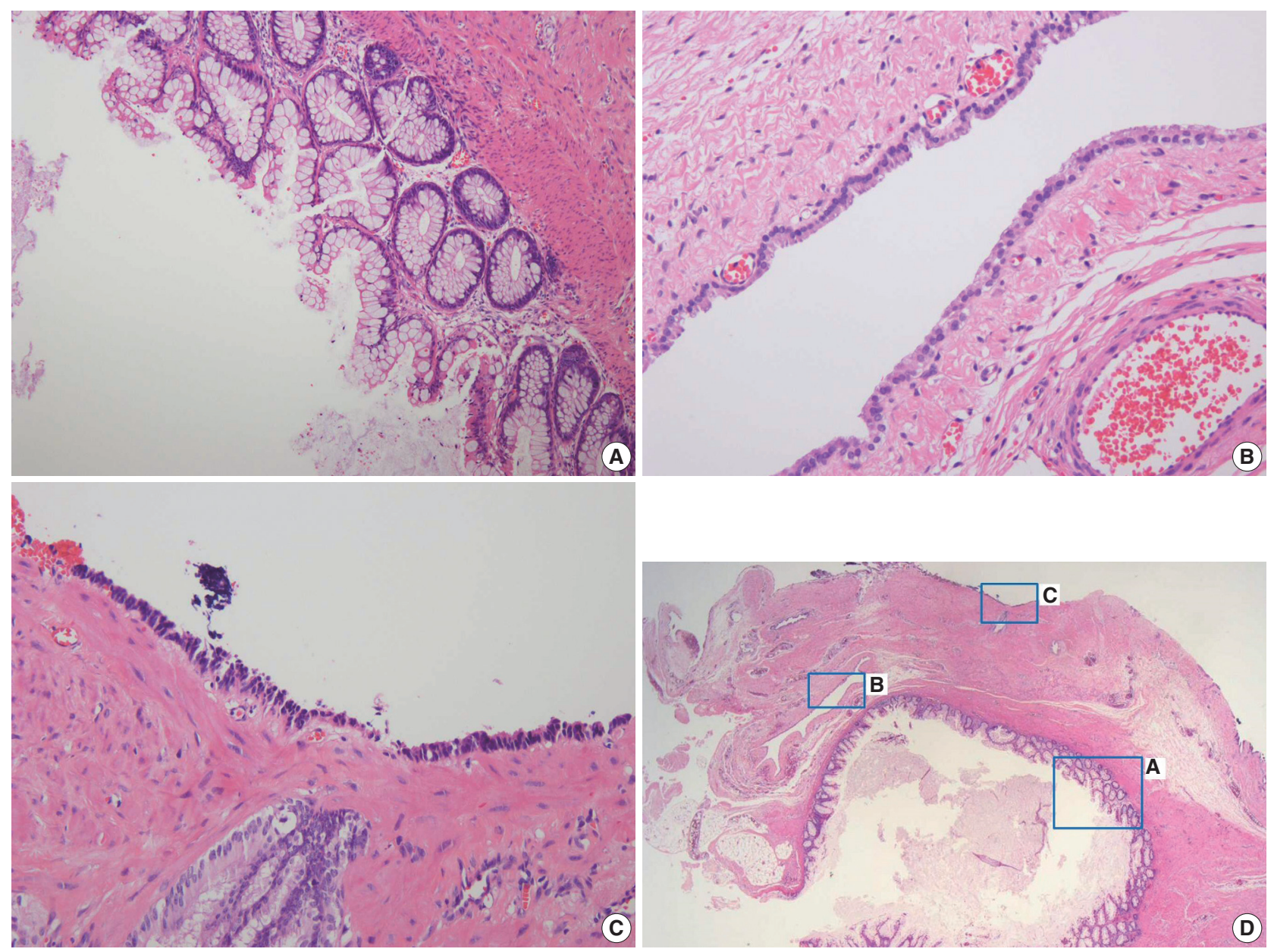

Fig. 2. On scanning view, different types of epithelia lining the cyst walls are identified (D). The cyst walls are lined by mucinous epithelium (A), low cuboidal epithelium (B), and pseudostratified columnar epithelium (C). 
sweat glands were found, similar to dermoid cysts. Enteric duplication cysts are lined with intestinal epithelia with typical villi and crypts, and contain well-defined layers of smooth muscle and the myenteric plexus. Gland cysts can be differentiated through their lower region, usually closer to the anal sphincter rather than the retrorectal space. Anterior meningoceles are related to a sacral defect. ${ }^{4}$

The diameter of tailgut cysts varies from 1 to $22 \mathrm{~cm}$, and they contain debris such as keratinous and mucinous material. According to Hjermstad and Helwig, ${ }^{3}$ tailgut cysts are usually multiloculated and lined by squamous transitional and glandular epithelia. Squamous epithelium, present in $75 \%$ of cysts, is the most common type, and $50 \%$ of the cysts were covered with ciliated columnar epithelia. Some cysts also have a metaplastic process secondary to inflammation. ${ }^{5}$ Malignant transformation is a rare complication of tailgut cysts. Only a few cases of adenocarcinomas, carcinoid tumors, neuroendocrine carcinomas, endometrioid carcinomas, adenosquamous carcinomas, squamous cell carcinomas and sarcomas have been reported.

To evaluate retrorectal masses, rectal examination, barium enema, transrectal sonography, computed tomography (CT), and MRI could be considered. Barium enemas and transrectal sonography are useful for initial viewing of the cystic nature of the masses. In particular, transrectal sonography demonstrates the unity of the layers of the rectum, whether cystic lesions are unilocular or multilocular, and also any internal echoes from mucoid materials or inflammatory debris. CT images of the tailgut cysts show well-defined, thin-walled, uni- or multilocular masses in the retrorectal region. ${ }^{6}$ Calcification is not a characteristic of these cysts, and if present, should prompt consideration of malignancy. MRI is the best modality for imaging tailgut cysts because of its good soft tissue contrast and multiplanar imaging capability, which are useful for determining surgical techniques.

Entire surgical excision of tailgut cysts is strongly recommended because of the possibility of recurrence, infection, and local dissemination of malignant cells. ${ }^{7}$ Various surgical approaches have been elaborated for removing tailgut cysts, including posterior, abdominal, and combined posterior and abdominal. ${ }^{8}$ The best excisional approach is dependent on the size and location of the mass, possibility of infection, and adherence to the sacral or pelvic walls. Usually, the posterior approach is recommended for small benign tailgut cysts below the level of the $\mathrm{S} 3$ vertebrae. If the lowest level of the tailgut cyst is at the $\$ 4$ vertebra, the mass should be approached through a transabdominal incision.

In conclusion, this rare case highlights that a tailgut cyst should be considered in the differential diagnosis of a sacrococcygeal mass in a neonate. Preoperative MRI is essential for planning the most appropriate surgical approach, and complete surgical excision is the treatment of choice for all tailgut cysts, as it provides definitive diagnosis, relieves symptoms, and prevents complications such as infection, fistula formation, and malignant degeneration.

\section{Conflicts of Interest}

No potential conflict of interest relevant to this article was reported.

\section{REFERENCES}

1. Prasad AR, Amin MB, Randolph TL, Lee CS, Ma CK. Retrorectal cystic hamartoma: report of 5 cases with malignancy arising in 2. Arch Pathol Lab Med 2000; 124: 725-9.

2. Al-Khuzaie J, Al-Hindi S, Hassan A, Al-Youssif R. Tailgut cyst in a newborn: report of a case and literature review. Bahrain Med Bull 2002; 24: 108-9.

3. Hjermstad BM, Helwig EB. Tailgut cysts: report of 53 cases. Am J Clin Pathol 1988; 89: 139-47.

4. Raisolsadat SM, Zabolinejad N, Tabrizian-Namini F, Faraji P. Tailgut cyst in an infant with imperforate anus: a case report. Iran J Pediatr 2013; 23: 597-600.

5. Mathieu A, Chamlou R, Le Moine F, Maris C, Van de Stadt J, Salmon I. Tailgut cyst associated with a carcinoid tumor: case report and review of the literature. Histol Histopathol 2005; 20: 1065-9.

6. Raje V, Raje V, Patil RK, et al. Tailgut cyst: a case report in a 9-monthold infant. Int J Surg Case Rep 2013; 4: 272-5.

7. Oh JT, Son SW, Kim MJ, Kim L, Kim H, Hwang EH. Tailgut cyst in a neonate. J Pediatr Surg 2000; 35: 1833-5.

8. Joyce EA, Kavanagh DO, Winter DC. A rare cause of low back pain: report of a tailgut cyst. Case Rep Med 2012; 2012: 623142. 